\title{
La bibliothèque nationale de l'avenir : quelques réflexions impertinentes
}

\section{The National Library of the Future: A few impertinent}

Thoughts

\section{La biblioteca nacional del futuro: algunas reflexiones impertinentes}

\section{Jean-Rémi Brault}

Volume 39, numéro 2, avril-juin 1993

Les bibliothèques nationales

URI : https://id.erudit.org/iderudit/1028744ar

DOI : https://doi.org/10.7202/1028744ar

Aller au sommaire du numéro

Éditeur(s)

Association pour l'avancement des sciences et des techniques de la documentation (ASTED)

ISSN

0315-2340 (imprimé)

2291-8949 (numérique)

Découvrir la revue

Citer cet article

Brault, J.-R. (1993). La bibliothèque nationale de l'avenir : quelques réflexions impertinentes. Documentation et bibliothèques, 39(2), 101-104.

https://doi.org/10.7202/1028744ar
Résumé de l'article

À partir de la notion proposée par l'UNESCO et généralement admise de ce que doit être une bibliothèque nationale, l'auteur réfléchit à ce qu'elle peut devenir durant les prochains siècles. Il passe en revue les principales fonctions de la bibliothèque nationale et essaie d'entrevoir dans quelle mesure et dans quel sens ces fonctions évolueront. Profondément convaincu du rôle essentiel d'une bibliothèque nationale dans la vie culturelle d'un État, l'auteur souhaite que ces institutions nationales s'orientent vers une meilleure utilisation de la technologie disponible.
Tous droits réservés (C) Association pour l'avancement des sciences et des techniques de la documentation (ASTED), 1993
Ce document est protégé par la loi sur le droit d'auteur. L'utilisation des services d'Érudit (y compris la reproduction) est assujettie à sa politique d'utilisation que vous pouvez consulter en ligne. 


\title{
La bibliothèque nationale de l'avenir : quelques réflexions impertinentes
}

\author{
Jean-Rémi Brault* \\ Montréal
}

À partir de la notion proposée par l'UNESCO et généralement admise de ce que doit être une bibliothèque nationale, l'auteur réfléchit à ce qu'elle peut devenir durant les prochains siècles. II passe en revue les principales fonctions de la bibliothèque nationale et essaie d'entrevoir dans quelle mesure et dans quel sens ces fonctions évolueront. Profondément convaincu du rôle essentiel d'une bibliothèque nationale dans la vie culturelle d'un État, l'auteur souhaite que ces institutions nationales s'orientent vers une meilleure utilisation de la technologie disponible.

\section{The National Library of the Future: A few Impertinent Thoughts}

Starting from the UNESCO proposition of what constitutes a national library and which has become generally accepted, the author reflects on what it could become in the centuries ahead. $\mathrm{He}$ summarises the major functions of a national library and speculates on how and in which direction these functions will evolve. Unshakeably convinced of the vital role a national library plays in the cultural life of a nation, the author hopes these national institutions will better use of available technologies.
La biblioteca nacional del futuro: algunas reflexiones impertinentes

A partir de la noción propuesta por la UNESCO, y generalmente aceptada, de lo que debe seruna biblioteca nacional, el autor hace una reflexión sobre su futuro en los próximos siglos. Hace una revisión de las principales funciones de la biblioteca nacional y trata de entrever hasta que punto y en que sentido evolucionarán estas funciones. El autor, muy convencido del papel esencial de una biblioteca nacional en la vida cultural de un estado, desea que estas instituciones nacionales se orienten hacia una mejor utilización de la tecnología disponible.
Que sera la bibliothèque nationale de l'avenir? celle du prochain millénaire? Voilà une question qu'il faudrait sans doute poser aux spécialistes de la futurologie ou aux personnes qui se reconnaissent certaines compétences en prospective. Audelà de toutes les spéculations plus ou moins farfelues, il est pourtant loisible de réfléchir de façon sérieuse et d'élaborer des hypothèses vraisemblables.

Retenons d'abord l'hypothèse de la viabilité de la bibliothèque nationale. L'attitude de l'être humain face au patrimoine documentaire sera révélatrice du niveau de son évolution culturelle. Mais il n'est pas raisonnable de penser qu'il refusera ce patrimoine, qu'il adoptera une vision négative à son égard, qu'il la négligera, voire qu'il l'abandonnera. Pourquoi ne le considérerait-il pas comme une étape vers une évolution toujours active, comme un témoin du passé qu'il a franchi et un stimulant du futur qui lui est offert et qui sera ce qu'il en fera. Il est même prévisible qu'il en fera ce que l'impulsion d'une société dont il a fait partie lui suggérera. Fernand Dumont écrit que «dans Le Capital, Marx a rappelé ce truisme: à la différence des abeilles, l'homme projette devant lui son édifice social». Et il explique: «Prenons le mot dans son double sens: de la société, tout homme se donne une projection et un projet ${ }^{1}$.

Ce projet de société reposera sûrement sur des changements inhérents à la culture et à l'évolution technologique, en même temps qu'il suscitera d'autres changements. Dans ce tourbillon, les bibliothèques en général, et les bibliothèques nationales en particulier, subiront à la fois des mutations et une évolution. Des mutations qui, par leurs caractéristiques de changements brusques, susciteront de nécessaires réactions d'évolution ${ }^{2}$. Mais le fait même qu'elles «mutent» et qu'elles "évoluent», signifie qu'elles existent. Clavel concluait: "Le XXle siècle s'ouvrira avec des bibliothèques vieux style, des institutions qui auront fait la première mutation [celle de l'automatisation], un petit groupe qui aura accompli la seconde [celle du disque optique]»3 ${ }^{3}$.

Donc, il nous apparaît raisonnable de prévoir que les sociétés du prochain millénaire voudront conserver des institutions qu'elles continueront peut-être d'appeler «bibliothèques nationales». II est également raisonnable de souhaiter que, globalement, «la bibliothèque nationale d'un pays [continuera d'être] celle qui est

\footnotetext{
* Jean-Rémi Brault a occupé le poste de conservateur en chef de la Bibliothèque nationale du Québec de 1974 à 1985 .

1. Fernand Dumont, Les idéologies, [Paris], Presses universitaires de France, 1974, p. 173.

2. Jean-Pierre Clavel, «La mutation» in Frans Vanwijngaerden et al., Liber amicorum Herman Liebaers, Bruxelles, 1984, p. 16.
}

3. Ibid., p. 22. 
chargée de rassembler et de conserver pour les générations futures tous les écrits produits dans ce pays ${ }^{4}$. Surtout, il est souhaitable que l'application de cette définition soit nuancée et adaptée au contexte temporel et technologique. L'administrateur général de la Bibliothèque nationale du Canada écrivait récemment: «Les bibliothèques nationales témoignent de l'histoire, des caractéristiques et des priorités des sociétés où elles naissent et se développent» ${ }^{5}$.

En premier lieu, il importe de préciser, voire de circonscrire, la notion des "écrits" à laquelle Sir F.C. Francis faisait référence. Or les «écrits» qui feront l'objet des préoccupations des bibliothèques nationales, et qui, à la vérité, sont d'ores et déjà inscrits dans les objectifs d'un grand nombre de bibliothèques nationales, ces "écrits» seront des "documents», qui incluront

toutes les informations verbales, visuelles, orales et numériques, sous la forme de cartes, de pages imprimées, de partitions, d'archives sonores, de films, de cassettes vidéo, de banques de données informatiques, bref, tout ce qui va de l'épigraphie aux techniques les plus avancées de discographie ${ }^{6}$.

Ce que seront les bibliothèques nationales du prochain millénaire? Comme toutes les bibliothèques, et encore davantage, elles seront des «multimédiathèques», ou, au moins, des médiathèques. Un peu d'audace permettrait de croire que, dans certains pays, elles abandonneront le nom de bibliothèque nationale pour coiffer celui de «médiathèque nationale»?.

D'ailleurs, depuis longtemps, les grandes bibliothèques nationales ont pris soin de recueillir des documents diversifiés qui témoignent de la vie culturelle de chaque pays et de chaque époque. Déjà, par exemple, la Bibliothèque nationale de Paris acquiert, outre les documents manuscrits et les documents imprimés, des estampes, des dessins, des affiches, des cartes postales, des photographies, des monnaies et des médailles, des disques et autres documents sonores, même des maquettes de décors et des costumes ${ }^{8}$. La venue de nouveaux types de «docu- ments» exigera de la part des bibliothèques nationales la volonté d'accueil qu'elles ont manifestée envers les documents dits traditionnels.

La présence de tous ces documents pose déjà, avec acuité, le problème de leur conservation. Et l'arrivée de nouveaux documents, comme par exemple, les documents sur des supports informatiques, ne simplifiera pas la tâche. Car, on sait combien paradoxal est le mandat assumé par les bibliothèques nationales: conserver durant une période «indéfinie» des documents conçus pour une durée limitée. Et, en même temps, communiquer aux lecteurs des documents dont la conservation exige mille précautions et un soin méticuleux. On a pu écrire avec raison que ce sont «deux devoirs jumeaux, contradictoires et qu'il faut pourtant concilier $)^{9}$.

Jusqu'ici, on s'est surtout préoccupé de la conservation des imprimés. De nombreuses enquêtes, de multiples études toutes plus élaborées les unes que les autres, des rapports de plus en plus provocants, ont étalé publiquement l'état lamentable de milliers, voire probablement, de millions de documents imprimés. Ces cris d'alarme ont suscité des actions salvatrices. Des mesures concrètes ont été prises. Le sauvetage de ce qui pourrait être récupéré a été amorcé. Surtout, la prise de conscience généralisée de ce grave problème s'inscrit dans le souci non moins généralisé de préservation du patrimoine culturel initié sous les supports.

Aussi, sans négliger la récupération ni la conservation des imprimés, l'attention des bibliothèques nationales

doit maintenant se tourner vers les autres supports: microfilms, microfiches, films, bandes magnétiques, disques optiques. Chacun de ces supports est soumis à son propre genre de détérioration et il nous fait recourir à différentes méthodes de conservation $^{10}$.

À cette fin, il sera important que les bibliothèques nationales fassent appel à tous les spécialistes disponibles, surtout ceux de l'entreprise privée, qu'au besoin ils mettent au point des projets conjoints pour contrer ce fléau et récupérer ce qui peut encore l'être.
En même temps, doit se poursuivre - et dans bien des cas s'amorcer - une efficace réflexion sur «les limites de la conservation». Faut-il conserver «tous» les documents? Faut-il conserver, par exemple, tous les documents dits éphémères, tous les documents publicitaires, toutes les parutions de tous les périodiques, journaux, revues, magazines, etc.? Sinon, où s'arrêtera le devoir de conservation? Quels seront les critères qui imposeront l'obligation de conserver certains documents et d'abandonner certains autres? Comment distinguer ceux-ci de ceux-là?

Le point de départ de cette réflexion peut fort bien se situer dans les notions de "contenu» et de «contenant», de «support original» et de «support de remplacement ou de conservation». Et, plus fondamentalement, de cette réflexion peut surgir des raisons exactes qui justifient la

4. F.C. Francis, «Organisation des bibliothèques nationales», in Colloque des bibliothèques nationales d'Europe, Vienne 8-27 septembre 1958, Tâches et problèmes des bibliothèques nationales, Paris, UNESCO, 1960, p. 13.

5. Marianne Scott, «Rétrospective... du bureau de l'Administrateur général», Nouvelles de la Bibliothèque nationale, vol. 25, no 1 (janvier 1993), 5.

6. D.F. McKenzie, La bibliographie et la sociologie des textes, [Paris] Éditions du Cercle de la Librairie, [1991], p. 31-32.

7. L'ancien administrateur général de la Bibliothèque nationale de Paris parlait dans le même sens. Voir: André Miquel, «Pour une mnémothèque nationale», Le Débat, no 48, 10.

8. Voir les statistiques des collections de cette institution dans: Bruno Blasselle et Jacqueline MeletSanson, La Bibliothèque nationale, mémoire de l'avenir, [Paris, Gallimard, 1990], p. 166-167.

9. Gilbert Ouy, «Les bibliothèques», in L'histoireet ses méthodes, sous la direction de Charles Samaran, [Paris, Gallimard, 1961], p. 113114 (Encyclopédie de la Pléiade, 11)

10. Marianne Scott, «Mettre les questions en perspective», in Prévenir ou guérir?: Actes $d u$ Colloque sur la conservation des collections: Montréal, 16-17 novembre 1989, organisé par le Groupe de travail sur la conservation des collections du Sous-Comité des bibliothèques, Conférence des recteurs et des principaux des universités du Québec, Montréal, Bibliothèque nationale du Québec, 1990, p. 29. 
conservation. Conserve-t-on l'exhaustivité du patrimoine documentaire, sous son supportoriginal, parceque la loi constitutive de la bibliothèque nationale l'a décrété à une époque où les supports de remplacement étaient des futuribles? Ne conservet-on pas ces documents parce que leur "contenu» témoignera d'une civilisation, de son état de développement?

Bien sûr, on le sait, la conservation du patrimoine documentaire est remise en question constamment, surtout par les pourvoyeurs de ressources financières, et d'une façon particulière durant les périodes de récession. Peut-on consacrer des sommes d'argent très importantes pour la préservation et la conservation des documents, alors qu'on ne peut assurer l'éducation de la population, qu'on ne peut fournir les services de santé essentiels aux citoyens, alors qu'en somme, on ne peut garantir le «primovivere». Ce raisonnement, ce questionnement, si primaire et si «politicien» qu'il paraisse aux yeux des professionnels du livre, est tout de même une réalité à laquelle il faut apporter des réponses.

Car, malgré tout, malgré les sophismes les plus raffinés, malgré toutes les nuances que ce précepte peut subir, rappelons avec force qu'il faut graver au fronton de toutes les bibliothèques nationales le devoir impératif de la conservation du patrimoine documentaire national. La réflexion sur les raisons qui imposent cette obligation doit inclure le fait que les chercheurs des prochains siècles voudront d'abord avoir accès au contenu beaucoup plus qu'à son contenant. Aussi, cette réflexion poussera ces mêmes chercheurs à se demander s'il est justifié de consacrer des milliers de mètres cubes à la difficile conservation des journaux, sous leur support original, alors qu'ils peuvent être miniaturisés, conservés en l'état de microfilms ou de microfiches, ou, encore mieux, sous le support des disques compacts. C'est l'éventuelle utilisation de ces documents, leur témoignage de la pensée à une époque et en un lieu donnés, qui justifie leur conservation et les crédits que requiert cette complexe opération. Or, il est évident que la conservation indéfinie des journaux et de nombreuses revues est pratiquement impossible et que la seule solution réside dans l'utilisation intelligente de la technologie disponible.
Tous ces efforts visant l'acquisition d'une collection nationale exhaustive et sa conservation ne peuvent se justifier que dans un contexte de diffusion. À cet égard, la rédaction et la mise en disponibilité d'une bibliographie nationale constitue pour les bibliothèques nationales un moyen de diffusion privilégié. Car, «elle reflète l'évolution des tendances culturelles et littéraires, les progrès du développement scientifique et économique, l'attention publique accordée à un événement social particulier»" ${ }^{11}$. À cette tâche essentielle, les bibliothèques nationales de l'avenir ne sauraient se soustraire. Tâche complexe, fort coûteuse, mais qu'elles sont seules, à l'intérieur d'un pays, à pouvoir et à devoir réaliser, puisqu'elles sont les seules à assurer l'exhaustivité d'une collection nationale.

Mais, il est probable que, dans ce secteur comme dans les autres, les bibliothèques nationales doivent modifier certaines façons de procéder. Ainsi, la mise en disponibilité de la bibliographie nationale sous le support papier pourrait bien disparaître. Déjà, la plupart des bibliographies nationales sont disponibles sous forme de banques de données informatisées. Et les organismes qui veulent $y$ avoir accès peuvent le faire facilement en utilisant la technologie déjà disponible. Les coûts relativement importants de l'impression de cette bibliographie pourrait être utilisés à d'autres fins, comme, par exemple, l'acquisition d'équipements technologiques appropriés.

Ce qui devra être modifié ou, au moins, considérablement amélioré, c'est la cohésion internationale qui doit inciter toutes les bibliothèques nationales au "partage des ressources», c'est-à-dire, au partage «sur une base de réciprocité [de] la collection des notices bibliographiques des publications de chaque pays ${ }^{12}$. La technologie actuelle, et très certainement, encore plus celle de l'avenir, ne peuvent que favoriser cette utilisation réciproque des travaux déjà réalisés. En somme, la mise en application du Contrôle bibliographique universel permettra aux bibliothèques nationales de remplir pleinement leur mandat, en fournissant «partout et rapidement, sous une forme internationalement acceptable, les renseignements bibliographiques essentiels relatifs aux publications monographiques publiées dans tous les pays ${ }^{13}$.
Donc, le rôle de diffuseur national qui doit être joué par la bibliothèque nationale ne saurait être pleinement réalisé sans une participation et une cohésion internationales. Et il trouvera un complément dans l'autre programme mis de l'avant par l'UNESCO, celui qui porte sur l'accessibilité universelle aux publications.

Tout se tient dans l'énorme mandat des bibliothèques nationales: acquisition exhaustive de la collection documentaire nationale, conservation, inventaire dans la bibliographie nationale qui sert la diffusion aussi bien sur le territoire national que dans l'ensemble des pays qui participent au programme proposé par les instances internationales. Les bibliothèques nationales de l'avenir vivront et se développeront dans la mesure où elles se révéleront indispensables dans leur pays respectif et dans le réseau international, dans la mesure où elles réaliseront le mandat qui leur a été confié par leur gouvernement respectif, mandat calqué sur le modèle proposé par l'UNESCO.

Or, leur rôle national consiste également à servir de «rassembleur» dans le processus de «planification systématique des services de bibliothèques d'un pays ${ }^{14}$. On ne dira jamais assez quel rôle essentiel les bibliothèques nationales pourraient jouer dans le développement cohérent d'un réseau de bibliothèques à l'intérieur d'un pays, si seulement, grâce à une volonté politique bien affirmée, on leur en donnait les moyens. À partir du moment où un État reconnaît "l'information comme un facteur primordial du développement national et l'obligation largement reconnue, par les États dans le monde, d'assurer à

11. Congrès international sur les bibliographiesnationales, Paris, 12-15 septembre 1977, La biblio graphie nationale: son rôle actuel et ses développements futurs, Paris, UNESCO, 1977, p. 10.

\section{Ibid., p. 45 .}

13. Margaret Wijnstroom, Rapport d'activités, juillet 1972-juillet 1973, présenté par la secrétaire générale au $39^{2}$ congrès de l'IFLA, Grenoble 1973,p. 4. (Document 112/F/PLE/8)

14. K.W. Humphreys, «Les fonctions d'une bibliothèque nationale",, Bulletin de l'UNESCOd l'intention des bibliothèques, vol. 20, no 4 (juilletaoût 1966), p. 171. 
tous les citoyens, indépendamment de leur condition sociale ou de leur milieu géographique, le plein exercice du droit à l'information $)^{15}$, à partir de ce moment-là, le même État prend les moyens concrets pour atteindre ses objectifs. Et il se trouve que la bibliothèque nationale peut devenir ce catalyseur des forces vives, apte à assurer ce développement d'un réseau cohérent, ou, au moins, à y participer très activement.
Ce que sera la bibliothèque nationale de l'avenir? Elle sera cette institution nationale qui devra continuer de se révéler un élément essentiel du développement culturel d'un État. Ouverte aux technologies les plus audacieuses, voire participant à leur progrès, accueillante pour tous les documents, porteurs d'un message, quel que soit son support, elle se situera dans une aire dynamique, refusant tout statisme stérilisant. Seules survivront les bibliothèques nationales qui auront refusé de se momifier ou, au moins, qui auront rapidement desserré leurs bandelettes.

15. Réal Bosa, «La planification nationale des services d'information documentaire", in Vers une planification des services québécois d'information documentaire; textes déposés lors de la Table ronde, 5 mai 1978, présentés par JeanRémi Brault, Montréal, ASTED, 1977, p. 56-57.

\section{INDEX DES ANNONCEURS}

Volume 39, numéro 2, 1993

Colette Rivet

Bibliothèque nationale du Canada

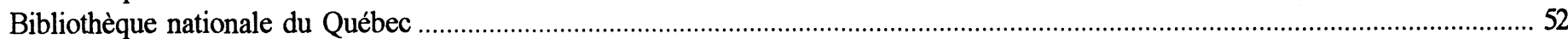

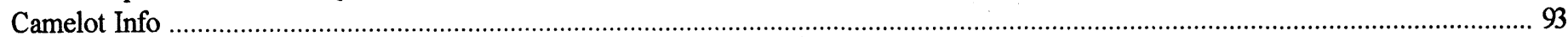

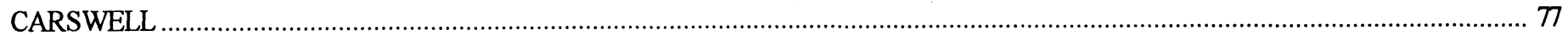

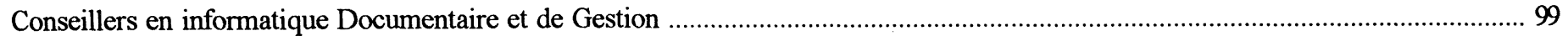

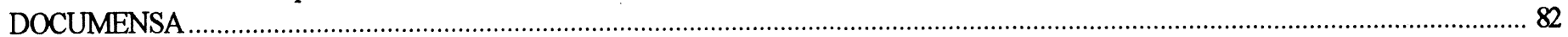

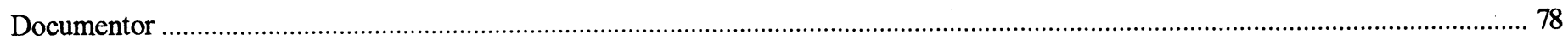

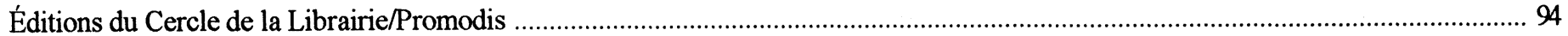

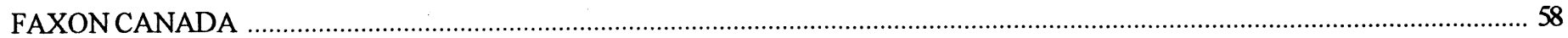

Gestion documentaire Best-Seller inc ...................................................................................................................... $2^{\mathrm{e}}$ couverture

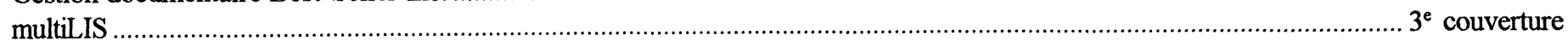

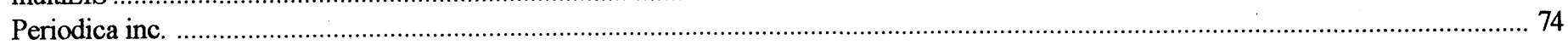

Les Services d'abonnement CANEBSCO ….......................................................................................................... $4^{\mathrm{e}}$ couverture

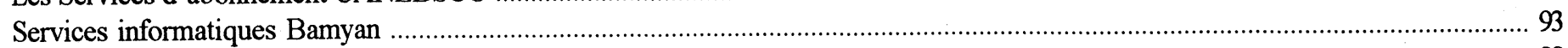

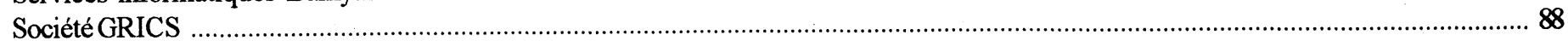

